\title{
Legen niedrige Zinsen den Keim für die nächste Finanzkrise?
}

\section{Linda Kirschner}

\section{Relevanz}

Eine expansive Geldpolitik soll Wirtschafts- und Finanzkrisen entschärfen und eine schnellere Erholung einleiten. Eine länger anhaltende Niedrigzinsphase entfaltet jedoch ungünstige Nebenwirkungen. Diese erschweren zunehmend den Ausstieg aus der Niedrigzinspolitik. Im Vergleich zu ihren Konkurrenten mit hohen Kapitalpolstern vergeben gerade die schlecht kapitalisierten Banken mehr und riskantere Kredite, die in der nächsten Rezession zu umso höheren Verlusten führen. Gerade die Banken mit wenig Eigenkapital können solche Verluste am wenigsten selber tragen und geraten leicht in existentielle Gefahr. Das könnte den Keim für die nächste Krise legen. Umso wichtiger wären ein rechtzeitiger und vorsichtiger Ausstieg aus der Niedrigzinsphase, ausreichende Kapitalstandards für Banken, sowie vorbeugende Massnahmen, welche die Risiken in der Realwirtschaft abbauen.

Christian Keuschnigg

\section{Quelle}

Der nachfolgende Text ist eine Zusammenfassung von: Jimenez, Gabriel, Steven Ongena, Jose-Luis Peydro und Jesus Saurina (2014), Hazardous Times for Monetary Policy: What do Twenty-three Million Bank Loans Say about the Effects of Monetary Policy on Credit Risk-taking? Econometrica 82, 463-505.

L. Kirschner $(\bowtie)$

Universität St. Gallen, St. Gallen, Schweiz

E-Mail: linda.kirschner@unisg.ch 
Spätestens seit der Finanzkrise von 2007-2009 wird darüber gestritten, ob ein niedriger Leitzins der Zentralbank die Risikobereitschaft von Banken erhöht. Dafür spricht, dass die Banken von 2002 bis 2005 zunehmend riskante Kredite vergaben und der Leitzins mehrheitlich auf einem niedrigen Niveau lag. Eine simple Korrelation ist aber noch kein Beleg für einen kausalen Zusammenhang. Es könnten auch andere makroökonomische Faktoren einschließlich niedriger langfristiger Zinssätze dafür verantwortlich sein. Die Geldpolitik braucht eine klare Antwort auf diese Frage, um in einer Finanzkrise richtig zu handeln, neuen Krisen vorzubeugen und ihre Folgen abzumildern.

Die Politik niedriger Leitzinsen, welche die kurzfristigen Zinsen senkt, kann das Risiko der Unternehmenskredite und im Nachgang den Umfang fauler Kredite auf zwei Wegen beeinflussen. Erstens bestehen bei niedrigen Zinsen auch viele eher knapp rentierliche Investitionsprojekte noch den Investitionstest der Unternehmen, die sich bei höheren Zinsen nicht mehr rechnen. Dazu kommt, dass niedrige Zinsen die Vermögenswerte steigen lassen, so dass der Wert der Sicherheiten und damit die Kreditfähigkeit scheinbar zunimmt. Wenn die Zinsen wieder ansteigen, sind viele Projekte nicht mehr rentabel und der Wert der Sicherheiten fällt. In der Folge steigt der Anteil fauler Kredite.

Zweitens gehen niedrige Zinsen auch mit hoher Liquidität einher. Daher können sich Banken sehr leicht kurzfristig und billig refinanzieren und das Volumen der Kreditvergabe ausdehnen. Dazu kommt, dass in einer Niedrigzinsphase sichere Anlagen wenig rentieren und die Banken auf der Suche nach Profitabilität ihre Mittel eher in riskante Unternehmenskredite anstatt in wenig rentierliche sichere Anlagen investieren.

Sowohl von der Seite der Unternehmen als auch der Banken steigern niedrige Zinsen nicht nur das Volumen der Unternehmenskredite, sondern auch ihr Risiko. Wenn nicht andere Vorkehrungen wie z. B. antizyklische Kapital- und Liquiditätsanforderungen bei den Banken greifen, kann also eine länger anhaltende Niedrigzinsphase den Keim für die nächste Finanzkrise legen. Der Beitrag der Forschungsarbeit ist es, den Einfluss niedriger Zinsen nicht nur auf das Volumen, sondern auch auf die Qualität der Kreditvergabe zu quantifizieren.

Die Forscher nutzen Daten des spanischen Kreditregisters, das detaillierte Informationen über sämtliche Kreditanfragen von Firmen sowie die Kreditentscheidungen der Banken und die Anforderungen an die Kreditsicherheiten enthält. Sie können auch die Zusammensetzung und den Umfang des Kreditportfolios der Banken beobachten. Der Datensatz umfasst die Zeitspanne von 2002 bis 2008 und reicht in die Zeit der großen Wirtschaftskrise hinein. Die Wahrscheinlichkeit einer Kreditzusage belief sich auf durchschnittlich $36 \%$. Neben den 
Daten des Kreditregisters nutzen die Ökonomen auch Bilanzdaten der jeweiligen Banken und Firmen. Die durchschnittliche Eigenkapitalquote der Banken lag bei eher geringen 5,5\%, ein Wert, der vor und während der Krise jedoch dem europäischen Durchschnitt entsprach. Das Risiko, dass Firmen ihren Zahlungsverpflichtungen nicht nachkamen, betrug durchschnittlich $3 \%$. Allerdings schwankte dieser Wert stark, einige Unternehmen besaßen also ein höheres Ausfallrisiko als andere. Ein Unternehmen galt als riskant, wenn es in den vergangenen 4 Jahren mindestens einmal zahlungsunfähig war.

Eine Senkung des Tagesgeldsatzes um einen Prozentpunkt steigert die Wahrscheinlichkeit einer Kreditzusage an zahlungssäumige Gläubiger durch schlechter kapitalisierte Banken um 3 Prozentpunkte mehr als bei stabilen Banken.

Konkret untersuchen die Forscher die Wahrscheinlichkeit einer Kreditzusage in Abhängigkeit der Veränderung des kurzfristigen Zinssatzes, der Eigenkapitalausstattung der Bank und des Risikos des Kreditnehmers. Die Analyse bestätigt zunächst die intuitive Vermutung, dass die Wahrscheinlichkeit einen Kredit zu erhalten, für ein riskantes Unternehmen geringer ist als für ein Unternehmen ohne auffällige Kredithistorie. Allerdings führt eine Reduzierung des Tagesgeldzinses um einen Prozentpunkt dazu, dass die Wahrscheinlichkeit einer Kreditzusage an ein riskantes Unternehmen um 7 Prozentpunkte steigt. Die Ergebnisse sind noch bedenklicher, wenn man die Kreditzusagen von Banken mit stabiler Eigenkapitalbasis mit den Entscheidungen gering kapitalisierter Banken vergleicht. Eine Bank mit wenig Eigenkapital hat weniger zu verlieren als eine vergleichbare Bank mit höheren Eigenmitteln. Schlecht kapitalisierte Banken sind deshalb bei sinkendem Zinssatz tendenziell stärker geneigt, riskantere Unternehmenskredite zu vergeben, die zwar öfter ausfallen, aber dafür im positiven Fall höhere Zinsen abwerfen. Die Autoren vergleichen jeweils das Verhalten von Banken, deren Eigenkapitalquote eine Standardabweichung (2 Prozentpunkte) auseinanderliegen. Abb. 1 zeigt den Unterschied in der Zunahme der Kreditvergabe in Abhängigkeit von der Eigenkapitalausstattung. Um den gezeigten Betrag nimmt die Wahrscheinlichkeit der Kreditzusage durch schlecht kapitalisierte Banken stärker zu als bei Banken mit höherem Eigenkapital. Dieser Unterschied zeigt sich nicht nur bei risikoarmen Unternehmen, sondern ist bei riskanten Unternehmen mit vergangenen Zahlungsausfällen sogar noch höher.

Zudem untersuchen die Autoren das Volumen der zugesagten Kredite und die mit der Kreditzusage verbundenen Anforderungen an Kreditsicherheiten. Es zeigt 


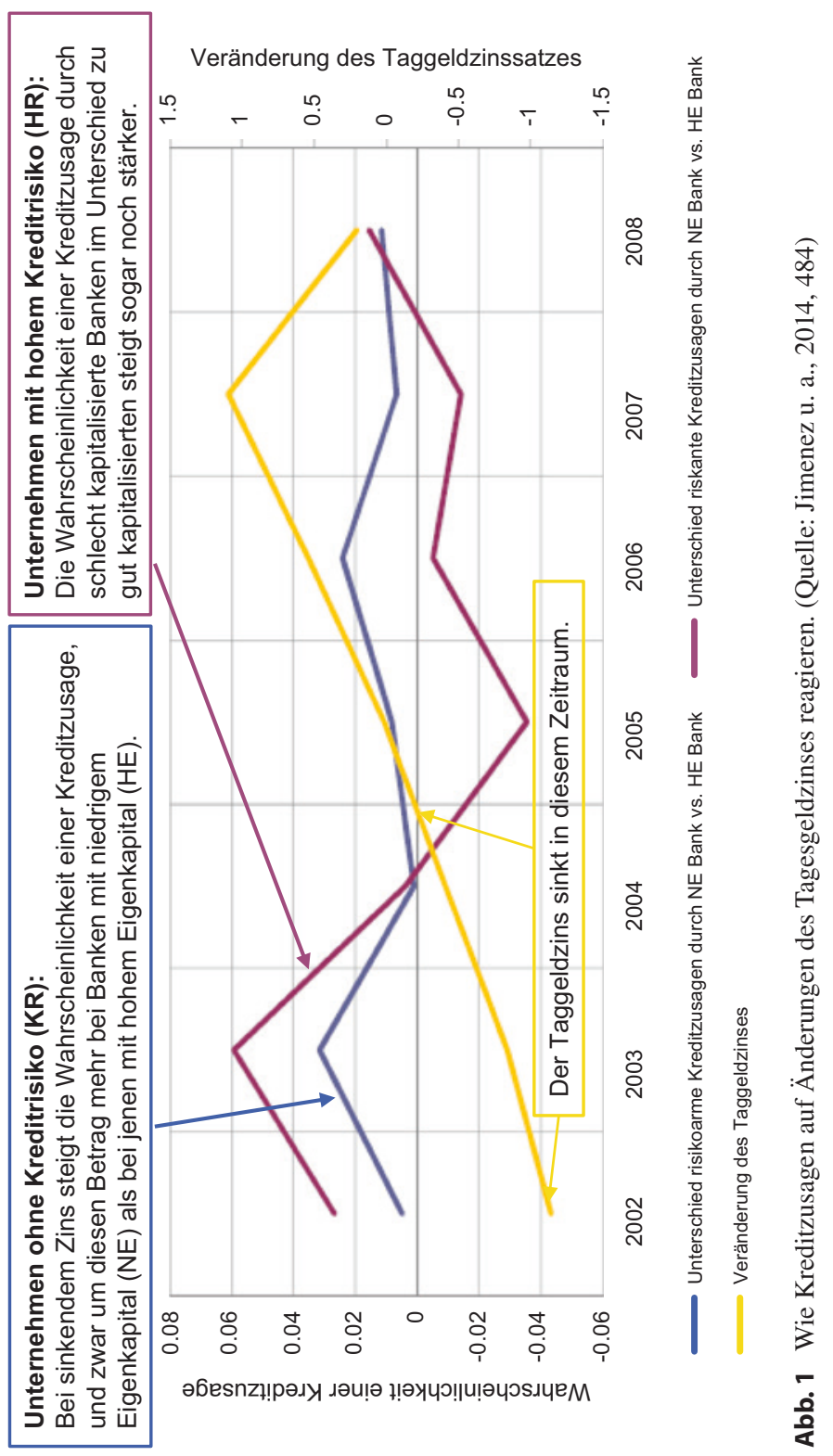


sich, dass Unternehmen, die in der Vergangenheit schon einmal zahlungsunfähig waren, kleinere Kreditbeträge erhalten als ihre Konkurrenten mit einer weißen Weste. Doch auch diese Situation ändert sich bei sinkenden Zinsen.

Wenn der Tagesgeldsatz um 1 Prozentpunkt sinkt, weiten schlechter kapitalisierte Banken ihr riskantes Kreditvolumen um $18 \%$ mehr aus als stabile Banken.

Niedrige Zinsen steigern das Kreditvolumen. Bei niedrigem Tagesgeldzins sagen die Banken mit höherer Wahrscheinlichkeit riskante Kredite zu und die versprochenen Kreditbeträge sind höher. Führt das zu einem Anstieg des Umfangs zukünftiger fauler Kredite? Das würde die nächste Finanzkrise begünstigen. Für eine Antwort sind die Kredite selbst, ihre Struktur und die zugrundeliegenden Sicherheiten eingehender zu betrachten. Nimmt nur das Kreditvolumen aufgrund gestiegener Liquidität zu oder gibt es eine strukturelle Verschlechterung in der Qualität der Kreditvergabe? Die Wissenschaftler untersuchen, ob die an riskantere Unternehmen vergebenen Kredite tatsächlich häufiger ausfallen und ob bei Zahlungsunfähigkeit weniger Sicherheiten zur Verfügung stehen, was die zu erwartenden Verluste der Banken steigert. Die Daten bestätigen die Vermutung. Die riskant vergebenen Kredite haben tatsächlich später eine höhere Ausfallwahrscheinlichkeit. Sie werden auch häufiger ohne Sicherheiten gewährt, sodass der Verlust für die Banken grösser wird.

Banken mit weniger Eigenkapital vergeben bei einer Senkung des Tagesgeldzinses riskantere Kredite, die mit einer um 5 Prozentpunkte höherer Wahrscheinlichkeit ausfallen. Gleichzeitig sinken die Anforderungen für die Besicherung. Die Wahrscheinlichkeit, dass diese Kredite gänzlich unbesichert bleiben, steigt im Vergleich zu stabilen Banken um fast 7 Prozentpunkte.

Ein niedriger Leitzins erhöht nicht nur das Kreditvolumen, sondern steigert auch das Risiko des Kreditportfolios und den Umfang der faulen Kredite. Besonders problematische Auswirkungen hat eine Niedrigzinsphase auf die Banken mit wenig Eigenkapital. Sie haben große Anreize zu riskantem Verhalten und können später kaum die Konsequenzen tragen, wenn das Risiko negativ ausschlägt. 
Open Access Dieses Kapitel wird unter der Creative Commons Namensnennung 4.0 International Lizenz (http://creativecommons.org/licenses/by/4.0/deed.de) veröffentlicht, welche die Nutzung, Vervielfältigung, Bearbeitung, Verbreitung und Wiedergabe in jeglichem Medium und Format erlaubt, sofern Sie den/die ursprünglichen Autor(en) und die Quelle ordnungsgemäß nennen, einen Link zur Creative Commons Lizenz beifügen und angeben, ob Änderungen vorgenommen wurden.

Die in diesem Kapitel enthaltenen Bilder und sonstiges Drittmaterial unterliegen ebenfalls der genannten Creative Commons Lizenz, sofern sich aus der Abbildungslegende nichts anderes ergibt. Sofern das betreffende Material nicht unter der genannten Creative Commons Lizenz steht und die betreffende Handlung nicht nach gesetzlichen Vorschriften erlaubt ist, ist für die oben aufgeführten Weiterverwendungen des Materials die Einwilligung des jeweiligen Rechteinhabers einzuholen.

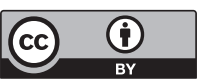

